\title{
A single amino acid substitution of the human immunodeficiency virus type 1 capsid protein affects viral sensitivity to TRIM5a
}

\author{
Ayumu Kuroishi' , Katarzyna Bozek2 Tatsuo Shioda' and Emi E Nakayama*1
}

\begin{abstract}
Background: Human immunodeficiency virus type 1 (HIV-1) productively infects only humans and chimpanzees but not Old World monkeys, such as rhesus and cynomolgus (CM) monkeys. To establish a monkey model of HIV-1/AIDS, several HIV-1 derivatives have been constructed. We previously reported that efficient replication of HIV-1 in CM cells was achieved after we replaced the loop between a-helices 6 and 7 (L6/7) of the capsid protein (CA) with that of SIVmac239 in addition to the loop between a-helices 4 and 5 (L4/5) and vif. This virus (NL-4/5S6/7SvifS) was supposed to escape from host restriction factors cyclophilin A, CM TRIM5a, and APOBEC3G. However, the replicative capability of $\mathrm{NL}-4 / 556 / 7$ SvifS in human cells was severely impaired.
\end{abstract}

Results: By long-term cultivation of human CEMss cells infected with NL-4/5S6/7SvifS, we succeeded in rescuing the impaired replicative capability of the virus in human cells. Sequence analysis of the CA region of the adapted virus revealed a G-to-E substitution at the 116th position of the CA (G116E). Introduction of this substitution into the molecular DNA clone of NL-4/5S6/7SvifS indeed improved the virus' replicative capability in human cells. Although the G116E substitution occurred during long-term cultivation of human cells infected with NL-4/5S6/7SvifS, the viruses with G116E unexpectedly became resistant to CM, but not human TRIM5a-mediated restriction. The 3-D model showed that position 116 is located in the $6^{\text {th }}$ helix near $L 4 / 5$ and $L 6 / 7$ and is apparently exposed to the protein surface. The amino acid substitution at the $116^{\text {th }}$ position caused a change in the structure of the protein surface because of the replacement of $\mathrm{G}$ (which has no side chain) with $\mathrm{E}$ (which has a long negatively charged side chain).

Conclusions: We succeeded in rescuing the impaired replicative capability of NL-4/5S6/7SvifS and report a mutation that improved the replicative capability of the virus. Unexpectedly, HIV-1 with this mutation became resistant to CM TRIM5a-mediated restriction.

\section{Background}

Human immunodeficiency virus type 1 (HIV-1) productively infects only humans and chimpanzees, but not Old World monkeys (OWM) such as cynomolgus (CM) and rhesus $(\mathrm{Rh})$ monkeys [1]. Unlike the replication of simian immunodeficiency virus isolated from macaques (SIVmac), HIV-1 replication is blocked early after viral entry, before the establishment of a provirus in OWM cells [13]. To establish a monkey model of HIV-1/AIDS, several viruses that are chimeras of HIV-1 and SIVmac (SHIV) have been constructed and tested for replicative capabil-

* Correspondence: emien@biken.osaka-u.ac.jp

1 Department of Viral Infections, Research Institute for Microbial Diseases, Osaka University, Osaka 565-0871, Japan

Full list of author information is available at the end of the article ity in simian cells $[4,5]$. The host range of HIV-1 was limited because of some intrinsic restriction factors in simian cells, such as ApoB mRNA editing catalytic subunit (APOBEC) 3G [6], cyclophilin A (CypA) [7-9], BST-2 (CD317; tetherin) $[10,11]$ and TRIM5 $\alpha$, a member of the tripartite motif (TRIM) family proteins [12]. Rh and CM TRIM5 $\alpha$ restrict HIV-1, but not SIVmac $[13,14]$. A lack of functional TRIM5 $\alpha$ expression in pig-tailed monkey enabled Hatziioannou et al. to construct a SHIV strain that differs from HIV-1 only in the vif gene and can efficiently replicate in pig-tailed monkeys [15]. Although this virus was designed to escape from monkey APOBEC3G mediated restriction, this virus failed to grow in $\mathrm{Rh}$ and CM cells. Kamada et al. attempted to evade the restrictions mediated by CypA in OWM cells by replacing the 
loop between $\alpha$-helices 4 and 5 (L4/5) of the HIV-1 capsid (CA) with that of SIVmac in addition to vif because CypA fails to bind to the L4/5 of SIVmac. However, this was not enough to escape from TRIM5 $\alpha$-mediated restriction [16].

TRIM $5 \alpha$ consists of RING, B-box 2, coiled-coil, and SPRY (B30.2) domains [17]. TRIM5 $\alpha$ recognizes the multimerized CA of an incoming virus by its $\alpha$-isoform specific SPRY domain [18-20]. Studies on chimeric TRIM5 $\alpha$ s have shown that the determinant of the species-specific restriction against viral infection resides in the variable regions of the SPRY domain [21,22]. On the other hand, we previously identified a single amino acid of the surface-exposed loop between $\alpha$-helices 6 and 7 (L6/7) of the HIV-2 CA as a determinant of the susceptibility of HIV-2 to CM TRIM5 $\alpha$ [23]. On the basis of this finding, we have succeeded in improving simian-tropic HIV-1, which was generated by Kamada et al. [5], by replacing L6/7 of CA with those of SIVmac239 in addition to L4/5 and vif [24]; the new resultant virus has more efficient replication in CM cells. The resultant virus, NL-ScaVR6/7S, showed efficient replicative capability in CM cells; however, the replicative capability of this virus in human cells was severely impaired.

In the present report, we describe our efforts to rescue the impaired replicative capability of NL-ScaVR6/7S after long-term cultivation in human CEMss cells, and we report on the amino acid mutation that improved the replicative capability of this virus.

\section{Materials and methods Viral adaptation}

For viral adaptation in human cells, $100 \mathrm{ng}$ of p24 of NLScaVR6/7S [24], renamed in this report as NL-4/5S6/ 7SvifS, was inoculated into $1 \times 10^{6}$ of human $\mathrm{T}$ cell line CEMss cells. The infected culture was gradually expanded to keep the cell concentration at $1 \times 10^{6} / \mathrm{mL}$. The culture supernatants were collected periodically, and p24 levels were measured with an ELISA kit (ZeptoMetrix, Buffalo, NY). Virus in the culture supernatant at day 42 after infection was designated NL-4/5S6/7SvifSd42, and inoculated into fresh CEMss cells. Six days after reinfection, the matrix (MA)-CA region of the integrated provirus was amplified by PCR from the genomic DNA of infected cells and cloned into PCR 2.1-TOPO vector (Invitrogen, Carlsbad, CA) to generate pTopo-MACAadp42. Nucleotide sequences of 6 independent clones were determined by ABI Prism 3100 Genetic Analyzer (Applied Biosystems, USA).

\section{DNA constructions}

The HIV-1 derivatives were constructed on a backbone of infectious molecular clone NL4-3 [25]. To introduce a glycine (G)- to-glutamic acid (E) substitution at the $116^{\text {th }}$ position of CA (G116E) into NL-4/5S6/7SvifS, the $0.5 \mathrm{~kb}$ SpeI-ApaI fragment, which corresponds to the $\mathrm{N}$-terminus of the CA including the 116th position and L6/7, of pTopo-MA-CAd42 was transferred into NL-4/5S6/7SvifS to generate NL-4/5SG116E6/7SvifS. The G116E substitution was also introduced into NL4-3 and NL-SVR (renamed NL-vifS in this report) by site-directed mutagenesis with the PCR-mediated overlap primer extension method. Resultant constructs were designated NL-G116E and NL-G116EvifS, respectively (Figure 1). To construct the wild type and mutant HIV-1 clones expressing green fluorescence protein (GFP), the $1.3 \mathrm{~kb}$ BssHII-ApaI fragment of NL-G116E, NL-4/5S6/7SvifS, or NL-4/ 5SG116E6/7SvifS, which corresponds to the MA and CA, was transferred to NL-Nhe GFP, in which the env gene was interrupted; and the GFP gene was inserted into the nef region. Resultant constructs were designated G116EGFP, 4/5S6/7S-GFP, and 4/5SG116E6/7S-GFP, respectively. To construct the lentivector expressing GFP under the control of cytomegalovirus promoter, we replaced the Eco RI-Apa I fragment corresponding to MA and CA of the pMDLg/p.RRE packaging vector $[24,26,27]$ with that of NL-G116E, and designated the resultant construct as pMDLg/p.RRE-G116E.

\section{Cells and virus propagation}

The human kidney adherent 293T cells were cultured in Dulbecco's modified Eagle medium supplemented with $10 \%$ heat-inactivated fetal bovine serum (FBS). The human T cell lines CEMss and MT4 were maintained in RPMI 1640 medium supplemented with 10\% FBS. Virus stocks were prepared by transfection of $293 \mathrm{~T}$ cells with HIV-1 NL4-3 and its derivatives using the calcium phosphate co-precipitation method. Viral titers were measured with an ELISA kit.

Sendai viruses $(\mathrm{SeV})$ expressing CM TRIM5 $\alpha$, human TRIM5 $\alpha$, Rh TRIM5 $\alpha$, and CM TRIM5 $\alpha$ without the SPRY domain [CM-SPRY (-)] were described previously $[18,23,28]$.

A cell line stably expressing CM or humanTRIM5 $\alpha$ was established as described previously [18]. Briefly, a pCEP4 plasmid (Invitrogen) encoding CM or human TRIM5 $\alpha$ fused with $\mathrm{HA}$ tag in its $\mathrm{C}$-terminus was transfected into TK-ts13 hamster cells. Transfected cells were then cultured in the presence of $0.3 \mathrm{mg} / \mathrm{ml}$ of hygromycin B (Gibco) for 14 days to remove untransfected cells. The expression of TRIM5 $\alpha$ was confirmed by Western blot analysis of cell lysate with anti-HA antibody (HA High Affinity, Roch).

\section{Viral infections}

CEMss or MT4 cells $\left(1 \times 10^{5}\right)$ were infected with $20 \mathrm{ng}$ of p24 of NL-4/5SvifS, NL-4/5S6/7SvifS, or NL-4/ 5SG116E6/7SvifS. The culture supernatants were collected periodically, and p24 levels were measured with an ELISA kit. To analyze the viral sensitivity to TRIM $5 \alpha, 1 \times$ 


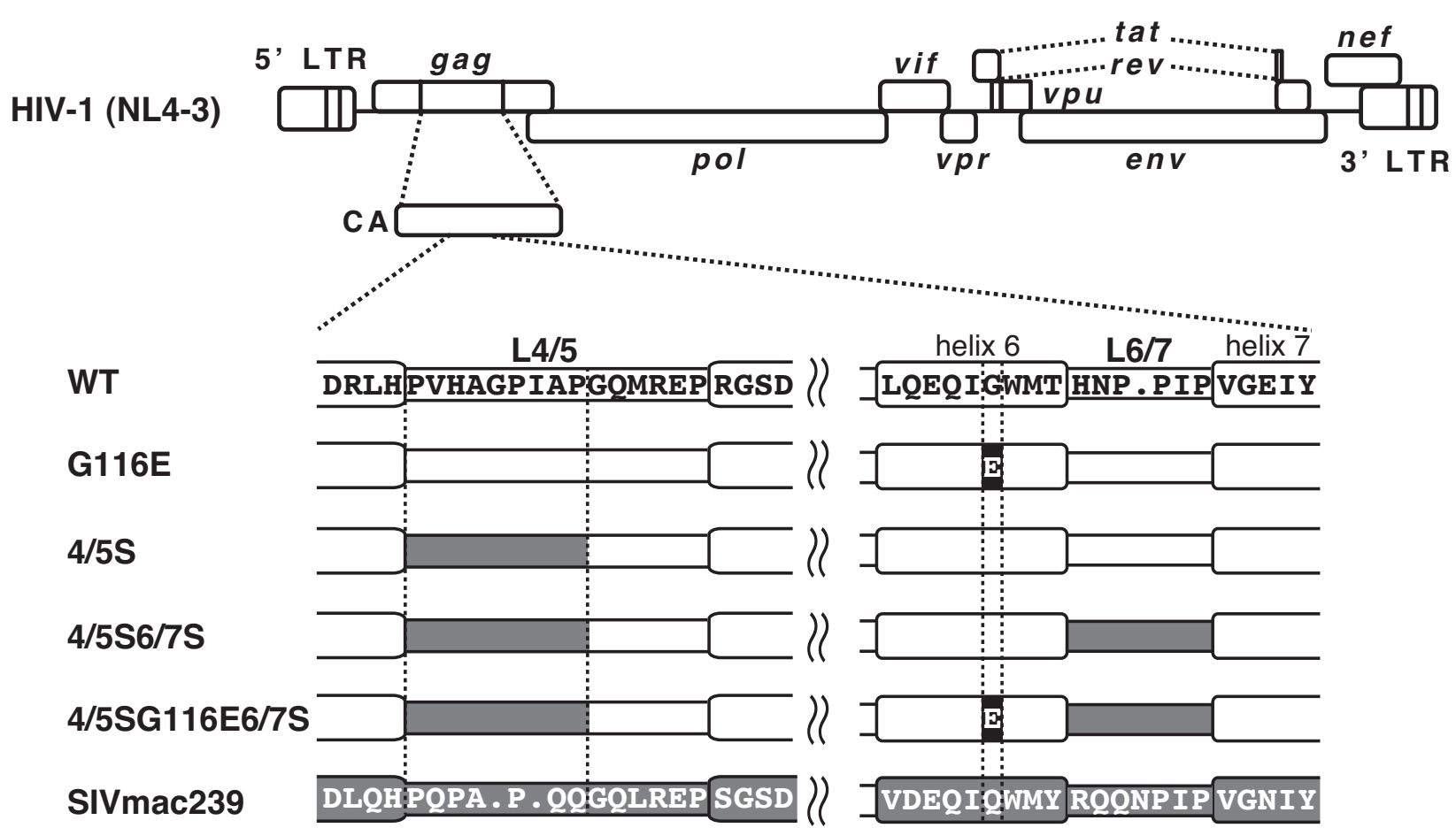

SIVmac239

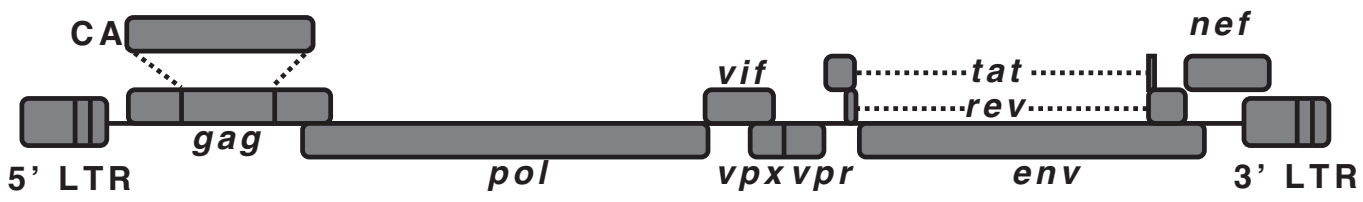

Figure 1 Schematic representation of HIV-1 derivatives. White and gray bars denote HIV-1 (NL4-3) and SIVmac239 sequences, respectively. "E" indicates the amino acid residue at the 116th position of the capsid protein (CA).

$10^{5}$ CEMss cells were first infected with SeV expressing each of the TRIM5 $\alpha$ s at a multiplicity of infection of 10 plaque-forming units per cell and incubated at $37^{\circ} \mathrm{C}$ for 9 hours. Cells were then superinfected with $20 \mathrm{ng}$ of p24 of HIV-1 NL4-3 or its derivatives. The culture supernatants were collected periodically, and the levels of p24 were measured with an ELISA kit.

For the single-round infection assay, CEMss or canine Cf2Th cells were infected with SeV expressing TRIM5 $\alpha$ as described above, and super-infected with vesicular stomatitis virus glycoprotein (VSV-G) pseudotyped HIV1 clones expressing GFP. In case of TK-ts13 hamster cells stably expressing CM, human or CM-SPRY(-) TRIM5 $\alpha$, cells were infected with VSV-G pseudotyped lentivector expressing GFP under the control of cytomegalovirus promoter. Two days after infection, the cells were fixed by formaldehyde, and GFP expressing cells were counted with a flow-cytometer. The percentage of the GFP-positive cells in the presence of TRIM5 $\alpha$ was divided by the percentage of GFP-positive cells in the presence of CMSPRY (-) to define the percent of infection. The differences in percent infection between WT-GFP and G116E-
GFP, or 4/5S6/7S-GFP and 4/5SG116E6/7S-GFP were statistically evaluated by using the unpaired test.

\section{Particle purification and Western blotting}

The culture supernatants of 293T cells transfected with plasmids encoding HIV-1 NL4-3 derivatives were clarified by low-speed centrifugation. Nine milliliters of the resultant supernatants were layered onto a $2 \mathrm{~mL}$ cushion of $20 \%$ sucrose in phosphate buffered saline (PBS) and centrifuged at 35,000 rpm for 2 hours in a Beckman SW41 rotor. After centrifugation, the virion pellets were resuspended in PBS, and p24 antigen concentrations were measured by ELISA. Fifty nanograms of p24 of HIV1 derivatives were applied to SDS-polyacrylamide gel electrophoresis, and the virion-associated proteins were transferred to a PVDF membrane. CA and CypA proteins were visualized with the anti-p24 antibody (Abcam) and anti-CypA antibody (Affinity BioReagents, Golden, CO), respectively.

\section{Modeling}

The structure of the N-terminal domain of the HIV-1 CA protein (PDB number 1GWP) [29] was used as a template for building the domain model with the G116E substitu- 
tion. The model was built using Modeller 9v4 [30] and visualized with PyMOL v1.0r2 (The PyMOL Molecular Graphics System, http://pymol.sourceforge.net/).

\section{Results}

A virus with SIVmac CA L4/5, $L 6 / 7$, and vif gained efficient replicative capability after adaptation in human $\mathrm{T}$ cell line

We previously reported that in addition to $\mathrm{L} 4 / 5$ of the CA and vif, L6/7 of the SIVmac CA is important for the efficient replication of HIV-1 derivatives in CM cells [24]. While introduction of SIVmac L6/7 into an HIV-1 derivative improved viral growth in $\mathrm{CM}$ cells, the replicative capability in human cells was greatly attenuated. To gain more insight into the effects of the L6/7 replacement on viral replication, we attempted to rescue the impaired replicative capability by long-term cultivation in human CEMss cells. NL-ScaVR6/7S, a virus with SIVmac L4/5, L6/7, and vif renamed NL-4/5S6/7SvifS in the present study, was inoculated into CEMss cells; and culture supernatants were periodically assayed for the levels of p24. Progeny virions were first detectable on day 20 after infection and reached a peak titer on day 42 (Figure 2A). The virus in the culture supernatant on day 42 was designated NL-4/5S6/7SvifSd42 and inoculated into fresh CEMss cells (Figure $2 \mathrm{~B}$ ). This time, the progeny virus was detectable on day 3 and reached a peak on day 20, suggesting that the NL-4/5S6/7SvifSd42 gained certain mutation(s) that overcame the attenuated replicative capability. Therefore, we amplified by PCR and cloned the integrated proviral DNA corresponding to the MA and CA regions in the NL-4/5S6/7SvifSd42-infected CEMss cells on day 6 . Nucleotide sequence analysis of the resultant clones revealed that 6 out of 6 independent clones carried a single nucleotide substitution at the 347th position of the CA region, resulting in a G-to-E substitution at the $116^{\text {th }}$ position of the CA (G116E).

Analysis of 95 HIV-1 strains in the Los Alamos HIV sequence databases http://www.hiv.lanl.gov/, including subtypes A to $\mathrm{K}$ of group $\mathrm{M}$, revealed that there was no HIV-1 strain carrying glutamic acid at the 116th position of the CA, although this position was occupied with variable amino acid residues (35 strains carried glycine; 36 , alanine; 9, threonine; 7, arginine; 6, glutamine; 1 each, isoleucine or aspartic acid).

\section{A single amino acid substitution in CA rescued impaired replicative capability in human cells}

To determine whether the single amino acid substitution at the 116th position of the CA improved the replicative capability of NL-4/5S6/7SvifS in human cells, we introduced the G116E mutation into NL-4/5S6/7SvifS. Resultant viruses were designated NL-4/5SG116E6/7SvifS and inoculated into human CEMss or MT4 cells together with their parental viruses to analyze their replicative
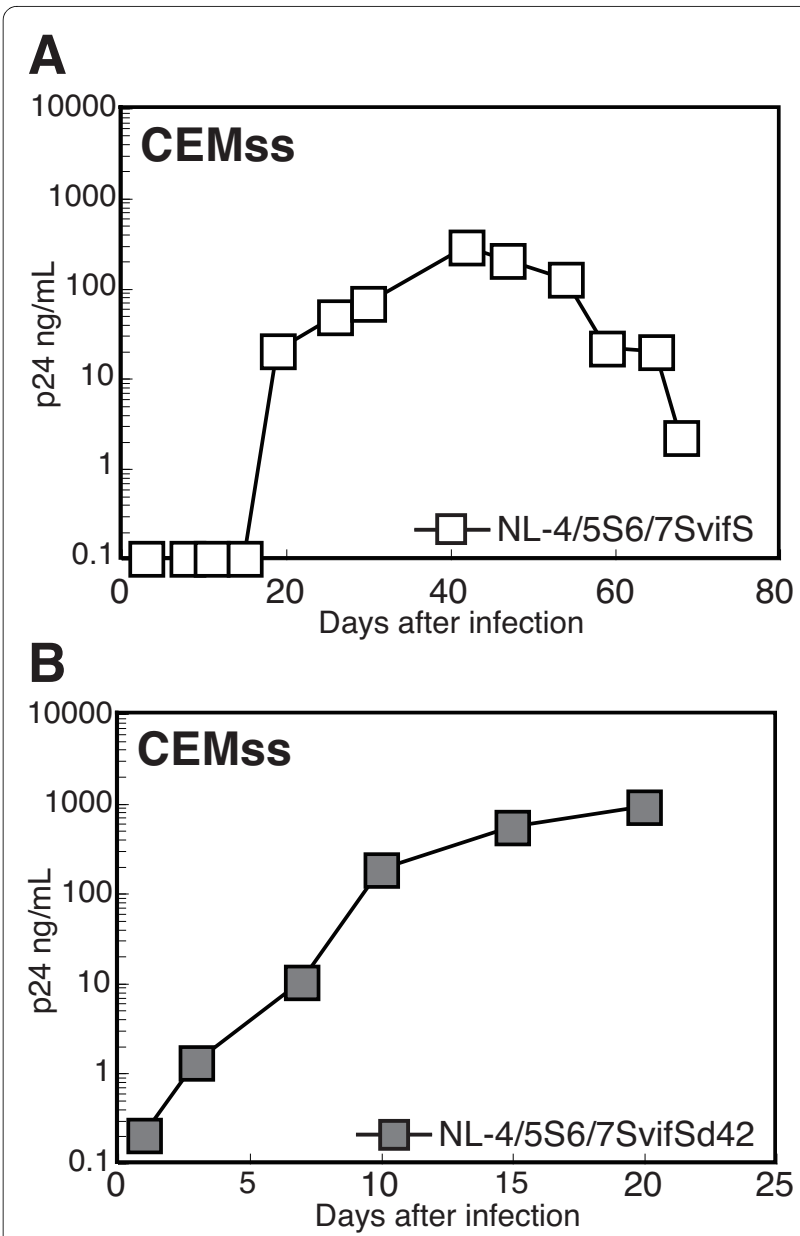

Figure 2 Adaptation of HIV-1 derivatives to human cells. (A) NL-4/ $5 S 6 / 7 S$ vifS, a virus with the SIVmac L4/5, L6/7, and vif was inoculated into CEMss cells, and culture supernatants were periodically assayed for the levels of p24. (B) Virus in the culture supernatant on day 42 after infection (NL-4/5S6/7SvifSd42) was inoculated into fresh CEMss cells.

capability (Figure 3). As described previously [24], NL-4/ 5S6/7SvifS showed less efficient growth in both CEMss and MT4 human cell lines than did NL-4/5SvifS. NL-4/ 5SG116E6/7SvifS could grow more efficiently in both human cells than did the parental NL-4/5S6/7SvifS, and its growth was comparable to that of NL-4/5SvifS (Figure 3 ). These data suggest that the rescued replicative capability of NL-4/5S6/7SvifSd42 in human cells (Figure 2) was the result, at least partly, of the acquisition of the G116E substitution in the CA.

The amino acid residue at the 116 th position of the CA affects viral growth in the presence of TRIM5a

We previously reported that NL-4/5S6/7SvifS could grow in CM cells [24], but failed to directly demonstrate that this virus could grow in human cells expressing $\mathrm{CM}$ TRIM5 $\alpha$ because of its impaired growth capability in human cells. Because the G-to-E substitution at the116th 

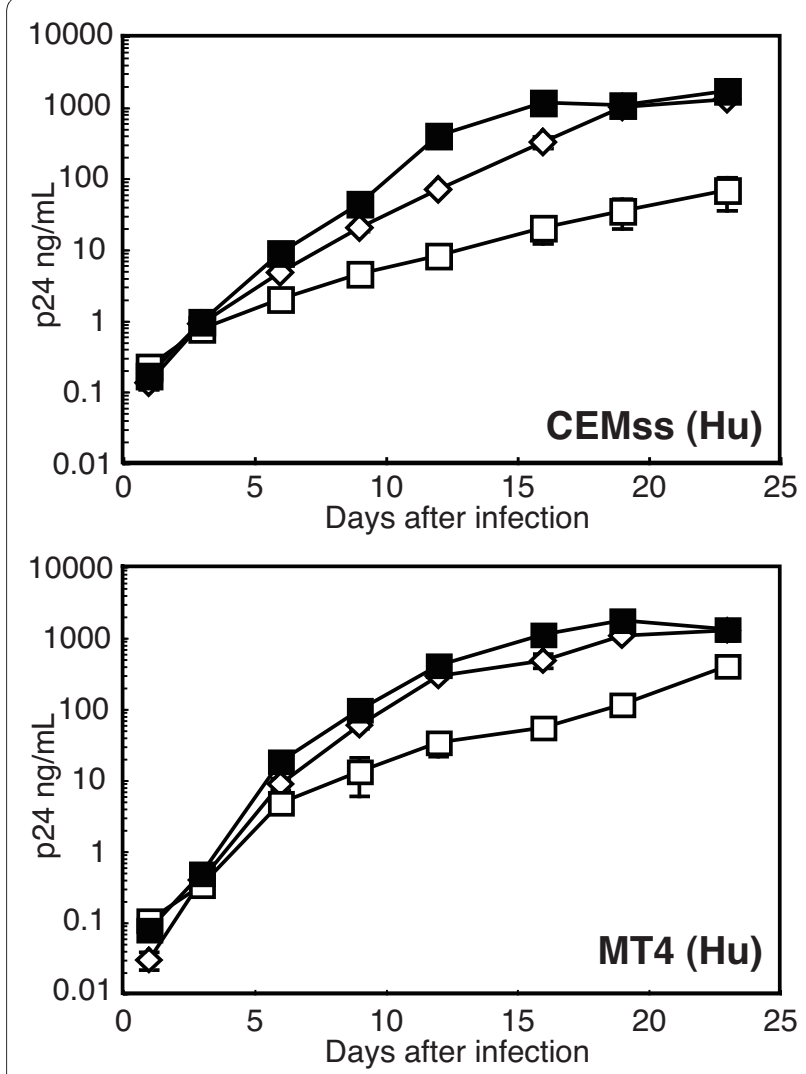

Figure 3 Replication properties of HIV-1 derivatives. Equal amounts of NL-4/5SvifS (white diamonds: virus with SIVmac L4/5 and vif), NL-4/5S6/7SvifS (white squares: virus with SIVmac L4/5, L6/7, and vif), or NL-4/5SG116E6/7SvifS (black squares: virus with the additional replacement of the 116 th amino acid Gly with Glu in NL-4/5S6/7SvifS) were inoculated into human CEMss or MT4 cells, and culture supernatants were collected periodically. The levels of p24 antigen were measured by ELISA. A representative of three independent experiments is shown.

amino acid position rescued the impaired growth capability of NL-4/5S6/7SvifS in human cells, we investigated whether NL-4/5SG116E6/7SvifS could grow in human cells expressing CM TRIM5 $\alpha$ (Figure 4A). For TRIM5 $\alpha$ expression, we used $\mathrm{SeV}$ expressing CM TRIM5 $\alpha$ or human TRIM5 $\alpha$. SeV expressing CM-SPRY (-) was used as a TRIM5 $\alpha$-negative control [31]. NL-SVR, a virus with SIVmac vif renamed NL-vifS in the present study, did not grow at all in CEMss cells expressing CM TRIM5 $\alpha$. In contrast, NL-4/5SG116E6/7SvifS could grow in CEMss cells expressing CM TRIM5 $\alpha$ (Figure 4A), although the viral titers were less than $10 \%$ of those in the absence of TRIM5 $\alpha$. Similarly, the human cell-adapted virus NL-4/ 5S6/7SvifSd42 could also grow in CEMss cells expressing CM TRIM5 $\alpha$ (data not shown). To clarify the impact of the single G-to-E substitution in CA on virus growth in

the presence of CM TRIM5 $\alpha$, we next introduced a G116E substitution in NL-vifS to generate NL-G116EvifS. We first anticipated that this virus would fail to replicate in CEMss cells expressing CM TRIM5 $\alpha$. Contrary to our expectations, however, this virus grew in the presence of CM TRIM5 $\alpha$ to levels similar to those of NL-4/ 5SG116E6/7SvifS. This result indicates that the single amino acid residue in CA could affect the viral sensitivity to CM TRIM $5 \alpha$ mediated restriction. To exclude any possible effect of SIVmac vif in NL-G116EvifS on TRIM5 $\alpha$ mediated restriction, we constructed NL-G116E, a virus with a single amino acid substitution at the 116th position of the CA only (Figure 4B). This virus could also replicate in CEMss cells expressing CM TRIM5 $\alpha$, confirming the importance of the 116th amino acid residue of the CA in TRIM $5 \alpha$-mediated restriction.

With respect to viral sensitivity to human TRIM $5 \alpha$, the growth of both NL-G116EvifS and NL-4/5SG116E6/ 7SvifS was slightly impaired compared with that of NLvifS in CEMss cells over-expressing human TRIM5 $\alpha$. The growth of the NL4-3 virus was not affected by human TRIM $5 \alpha$, while that of NL-G116E was slightly suppressed by human TRIM $5 \alpha$. These results suggest that the viruses with G116E substitution were more sensitive to human TRIM5 $\alpha$ although the G116E substitution occurred during long-term cultivation of human cells infected with NL-4/5S6/7SvifS. This excludes a possibility that the improved replicative capability of human cell-adapted virus is the result of escape from human TRIM $5 \alpha$-mediated restriction.

\section{A G116E substitution affects viral sensitivity to CM TRIM5a- mediated restriction in a single-round infection assay}

The assay described in Figures 3 and 4 investigated the effects of CM TRIM5 $\alpha$ on the multi-step growth of the viruses. To evaluate the effects of CM TRIM $5 \alpha$ on the early steps of viral infection, we performed a single-round infection assay. The fragment of NL-G116E, NL-4/5S6/ 7SvifS, or NL-4/5SG116E6/7SvifS corresponding to the MA and CA was transferred to an env-deleted HIV-1 genomic clone, which express GFP after infection. VSV-G pseudotyped wild type and mutant HIV-1 GFP viruses were inoculated into CEMss cells expressing TRIM5 $\alpha$ and GFP positive cells were counted 2 days after infection (Figure 5A). Because the replicative capability of NL-4/ 5S6/7SvifS in human cells was lower than that of the wild type virus as described above, it was highly likely that the infectivity of 4/5S6/7S-GFP would also be lower than those of WT-GFP and G116E-GFP. Therefore, we used higher input doses of 4/5S6/7S-GFP and 4/5SG116E6/7SGFP than those of WT-GFP and G116E-GFP. Ratios of the GFP-positive percentage of cells expressing CM TRIM5 $\alpha$ to those of cells expressing non-functional CMSPRY(-)-TRIM5 $\alpha$ are shown as percent of infection in 

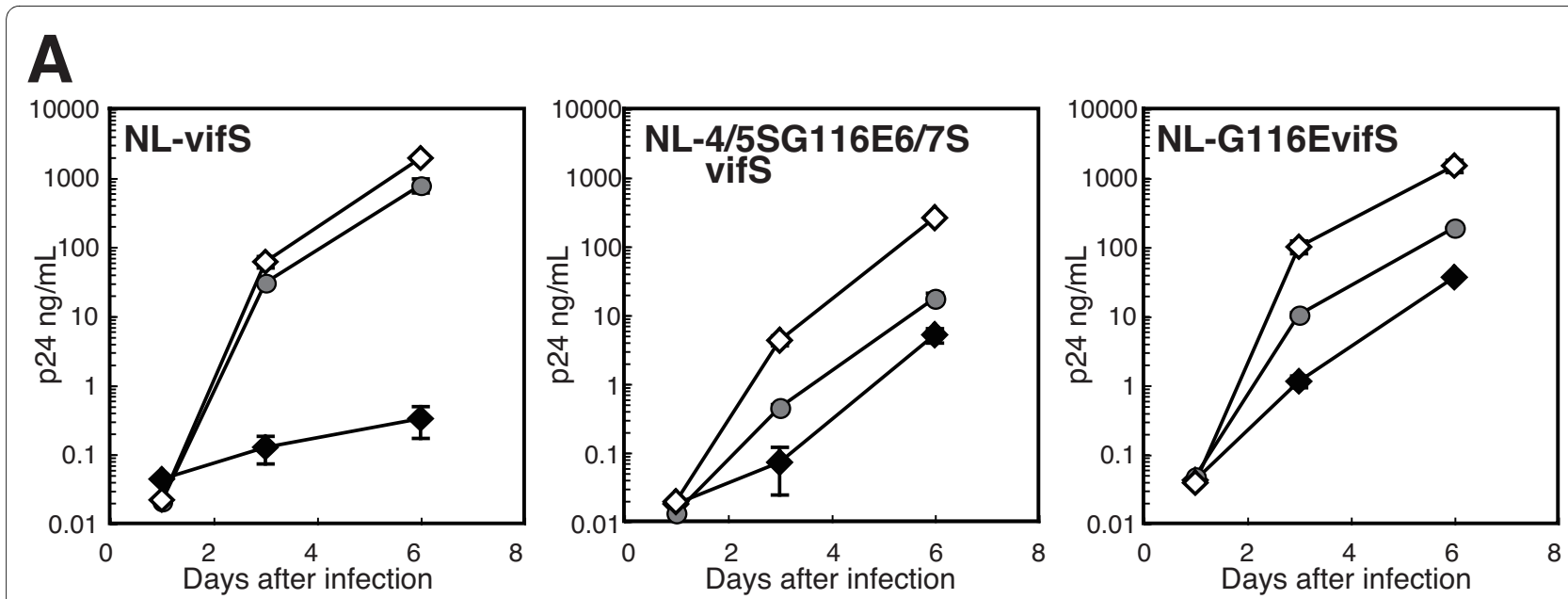

- CM TRIM5 $\alpha-$ - Hu TRIM5 $\alpha \prec$ CM SPRY(-) TRIM5 $\alpha$

B

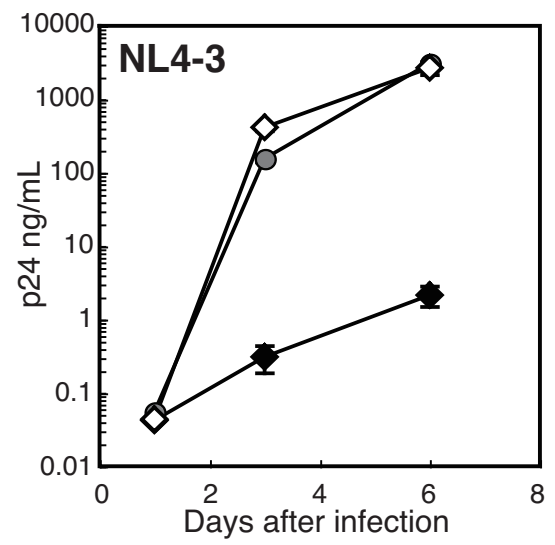

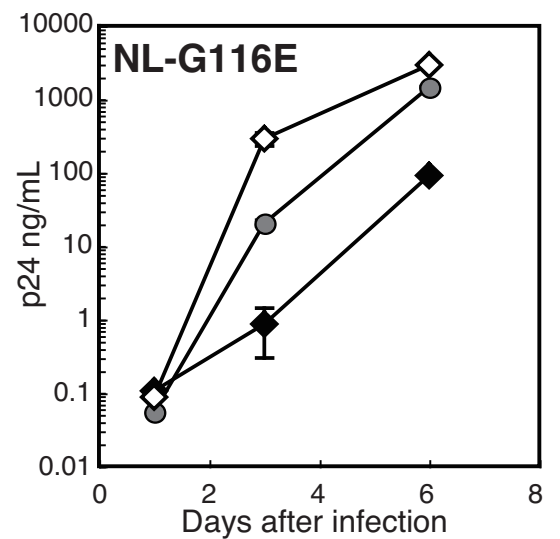

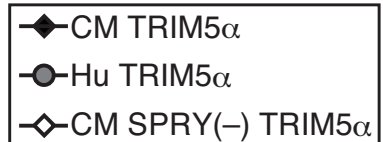

Figure 4 Viral growth in the presence of TRIM5a. CEMss cells were infected with recombinant Sendai virus (SeV) expressing CM (black diamonds), human (gray circles), or CM-SPRY (-) (white diamonds) TRIM5a. Nine hours after infection, cells were superinfected with the indicated HIV-1 derivatives. Culture supernatants were separately assayed for levels of p24. Error bars show actual fluctuations between levels of p24 in duplicate samples. A representative of three independent experiments is shown.

Figure 5B. The percent of infection was relatively constant among the different input doses. Consistent with the results that NL-G116E could replicate in human cells expressing CM TRIM5 $\alpha$ (Figure 4B), the GFP-expressing virus with the G116E substitution was more resistant to CM TRIM5 $\alpha$-mediated restriction than the wild type virus, while both viruses were completely restricted by $\mathrm{R} h$ TRIM5 $\alpha$ (Figure 5A, Figure 5B left). Similar results were obtained when we used Cf2Th canine cells lacking endogenous TRIM5 $\alpha$ expression, although the number of GFP-positive cells was less than that of CEMss cells (data not shown). These results in the single-round infection assay clearly confirmed our results in the live virus replication experiments showing that the G116E substitution conferred resistance against CM-TRIM5 $\alpha$-mediated restriction. While both the GFP-expressing viruses with the $4 / 5 \mathrm{~S} 6 / 7 \mathrm{~S}$ (4/5S6/7S-GFP and 4/5SG116E6/7S-GFP) were resistant to CM TRIM5 $\alpha$, an additional effect of the G116E substitution was not observed (Figure 5B, left). To examine the effect of G116E substitution in cells with more physiological levels of TRIM $5 \alpha$ expression, we established TK-ts13 hamster cells stably expressing CM or human TRIM5 $\alpha$ and inoculated lentivector expressing GFP under the cytomegalovirus promoter into these cells. As shown in Figure 5C and 5D, the GFP expression from the lentivector with the wild type CA was suppressed in TK-ts13 cells expressing CM TRIM5 $\alpha$, although the levels of suppression were less than those in Figure 5B due to lower levels of CM TRIM $5 \alpha$ expression. As expected, the lentivector with the G116E substitution showed reduced suppression by CM TRIM $5 \alpha$ compared with the wild type CA (Figures 5C and 5D). 


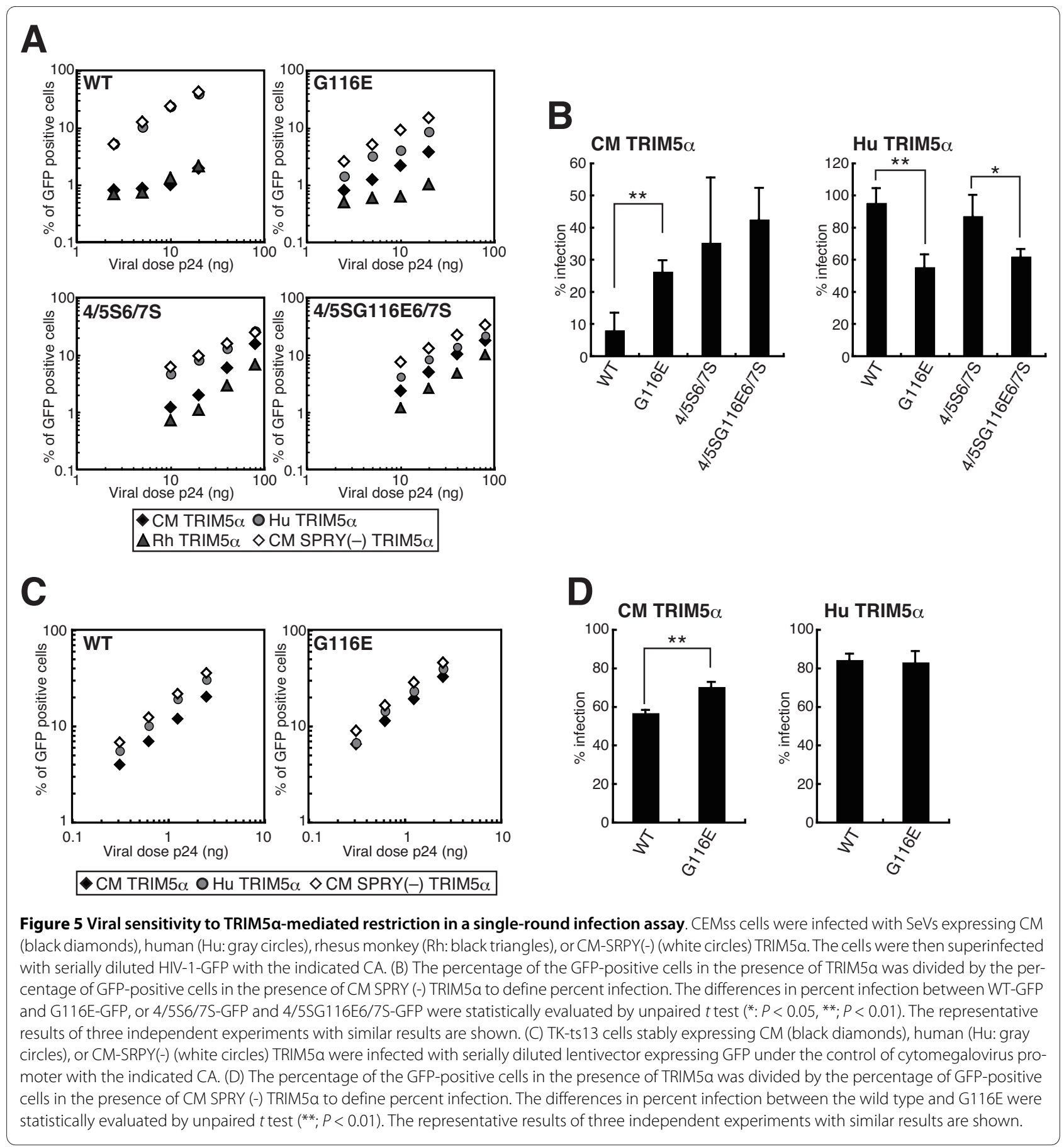

On the contrary, the GFP-expressing virus with G116E was more sensitive to humanTRIM5 $\alpha$ expressed from the $\mathrm{SeV}$ in CEMss cells than the wild type virus (Figure 5B, right). These results again confirmed the results in the live virus replication experiments shown in Figure 4. In the case of TK-ts13, cells stably expressing human TRIM5 $\alpha$ in which TRIM5 $\alpha$ expression is in more physiological levels; however, the difference in sensitivity to human TRIM5 $\alpha$ between the wild type and G116E lentivector was not observed (Figure 5C and 5D). Furthermore, when we used TRIM5 $\alpha$ knockout Jurkat cells, we also failed to detect the difference in sensitivity to human TRIM $5 \alpha$ between the wild type and G116E virus (data not shown). These results indicated that the effect of G116E substitution is virtually negligible at physiological levels of endogenous human TRIM $5 \alpha$, although this sub- 
stitution increases the susceptibility of HIV-1 to human TRIM $5 \alpha$.

\section{A G-to-E substitution at the 116th position did not affect the association between CA and CypA or Gag processing} To clarify whether the 116th amino acid substitution affects the association of CypA with CA, the CypA content in the wild type and mutant virions was evaluated by Western blot analysis. As shown in Figure 6, CypA was detected in virions with HIV-1 L4/5 (lanes 1 to 4, upper panel), but not in those with SIVmac L4/5 (lanes 5 to 7) indicating that the G-to-E substitution at the 116th amino acid position had no effect on CypA binding of HIV-1 CA. When we used anti-p24 antibody (Figure 6, lower panel), p55 Gag precursors and mature p24 CA were detected. The HIV-1 Gag precursor proteins with SIVmac L4/5 and L6/7 were processed nearly normally in the virion, although there were slight differences in the ratios of p24 to p55 among HIV-1 derivatives (Figure 6, bottom). In particular, the virus with SIVmac L4/5 and L6/7 tended to contain increased amount of p55 Gag precursors (lane 6, bottom); however, addition of G116E substitution did not facilitate the cleavage of Gag (lane 7).

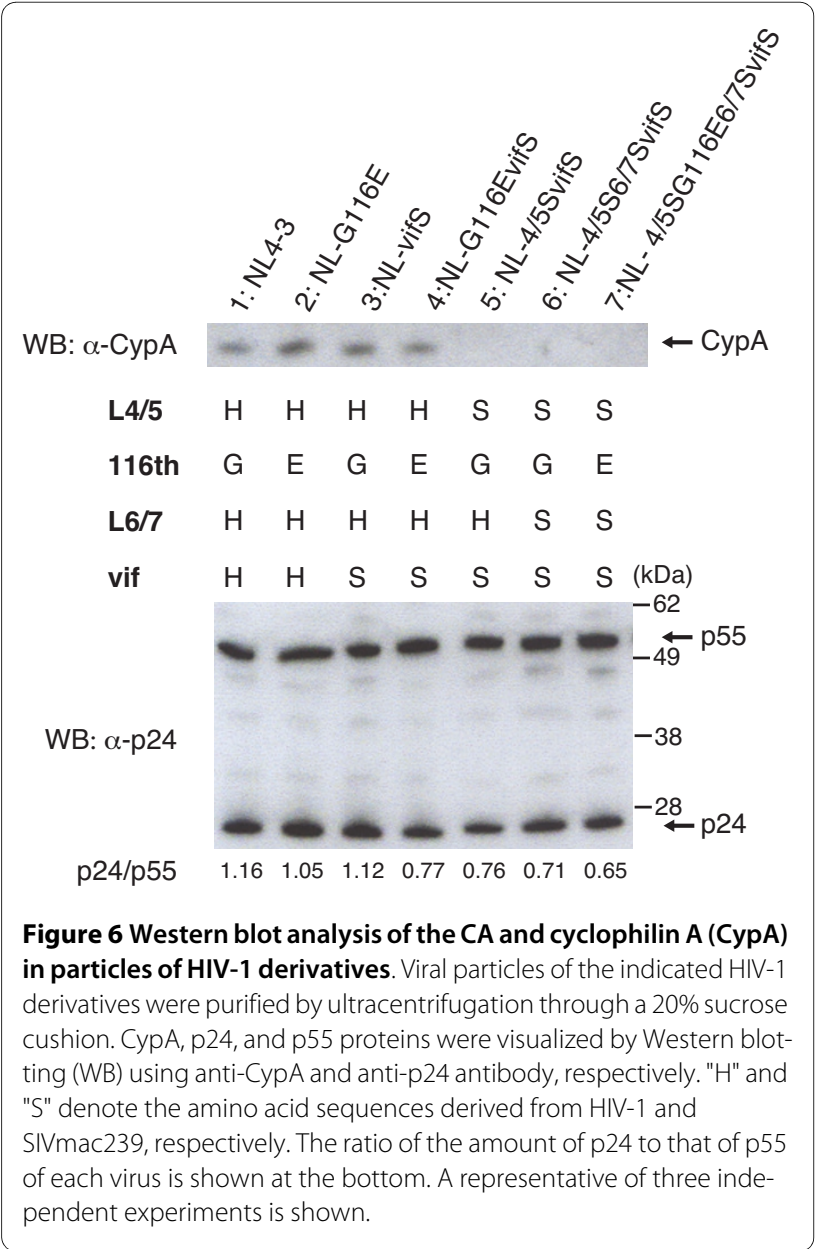

\section{Structural model of the capsid protein}

To obtain further insight into the effects of the G-to-E single amino acid substitution at the 116th position of the CA on its three-dimensional (3-D) structure, the 3-D model of the $\mathrm{N}$-terminus of the $\mathrm{CA}$ was constructed by homology-modeling on the basis of the published crystal structure of the N-terminus of the CA of NL4-3 (PDB number 1GWP) [29] (Figure 7). Position 116 is located in the $6^{\text {th }}$ helix near the L4/5 and L6/7 and is apparently exposed to the surface of the protein (Figure 7 upper panels). The substitution of $\mathrm{G}$ to $\mathrm{E}$ might be important because in contrast to $G$, which lacks a side chain, $E$ has a long side chain with a negative charge (Figure 7 lower panels). The mutation can therefore have two possible effects. First, if the residue is located in the interaction site, it can change the local complementarity between CA and TRIM5 $\alpha$. Second, even if the residue is not directly in the binding site, the change in the side chain and polarity can influence the configuration of nearby loops and, thereby, influence a binding site that is located somewhere else on the protein. Notably, the loops being flexible parts of the protein are slightly repositioned in the modeled structure with G116E substitution (Figure 7A and $7 \mathrm{~B})$.
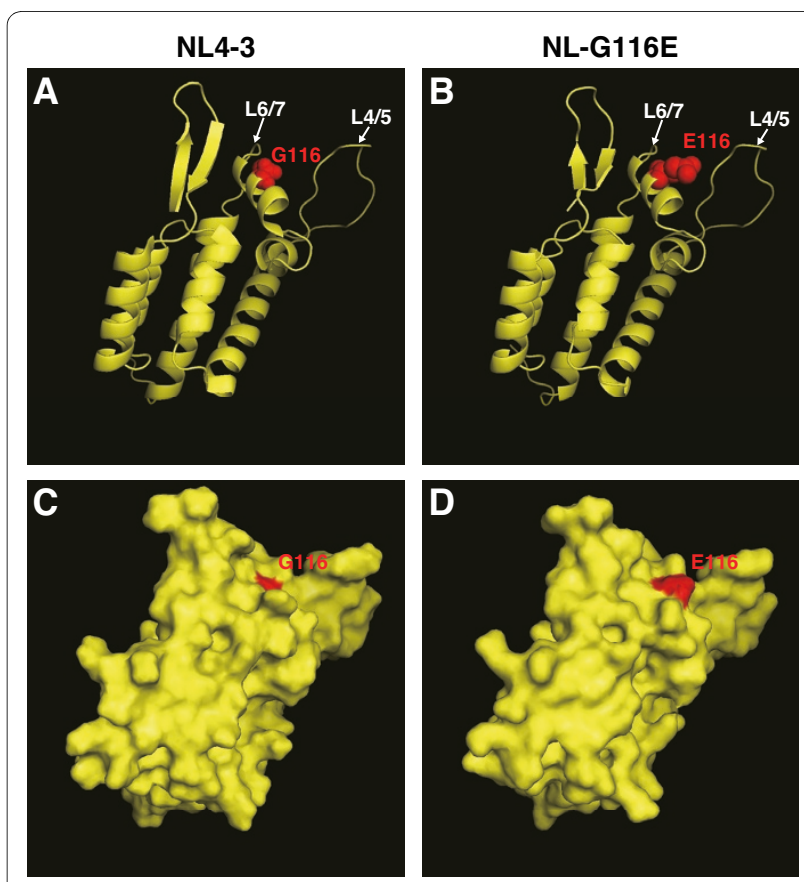

Figure 7 Structural model of the N-terminal domain of HIV-1 CA with $\mathrm{G} 116 \mathrm{E}$ substitution. Panel $A$ shows the template structure of the $\mathrm{N}$-terminal domain of the HIV-1 CA; panel B shows the model of the domain structure with the G116E mutation. The ribbons represent the protein backbone; $G$ and $E$ on the $116^{\text {th }}$ position with their side chains are shown in red spheres. Panels $C$ and $D$ show surface views of the template and model structures respectively with the $116^{\text {th }}$ position indicated in red. The loops between a-helices 4 and $5(L 4 / 5)$ and 6 and 7 $(\mathrm{L} 6 / 7)$ are labeled. 


\section{Discussion}

By long-term cultivation of human CEMss cells infected with NL-ScaVR6/7S (NL-4/5S6/7SvifS), a simian tropic HIV-1 that could grow efficiently in CM cells but inefficiently in human cells, we succeeded in rescuing the impaired replicative capability of the virus in human cells. Sequence analysis of the MA-CA region of the adapted virus revealed that the there was a G-to-E single amino acid substitution at the 116th position of the CA. Introduction of this substitution into the molecular DNA clone of NL-4/5S6/7SvifS indeed improved the virus' replicative capability in human cells. We thus concluded that the recovered replicative capability in human cells was mainly the result of acquisition of the single amino acid substitution at the 116th position of the CA, although small effects of mutations in regions other than the MACA cannot be fully excluded at present.

Although the 116th position of the CA is highly variable among natural HIV-1 strains from subtypes A to K, no virus with $E$ at the 116th position was found in the Los Alamos HIV sequence database 2009 http:// www.hiv.lanl.gov/. On the other hand, most HIV-2 and SIVmac strains have glutamine, which has a long side chain similar to E, at this position, and some strains have E. It is possible that the combination of the amino acid residue at the 116th position and L6/7 is important for viral growth. Consistent with this hypothesis, NL-4/ 5SG116EvifS, a virus with an HIV-1 derived L6/7 and the G116E substitution, showed impaired growth in MT4 cells (data not shown).

The precise reasons for the impaired replicative capability of NL-4/5S6/7SvifS and effect of G116E in human cells remain to be elucidated. Analysis of a series of CA mutants shown in Figures 4 and 5 clearly excluded the possibility that the impaired replicative capability of NL4/5S6/7SvifS in human cells resulted from an increased sensitivity to human TRIM $5 \alpha$ because a virus with the SIVmac L4/5 and L6/7 (4/5S6/7S) showed similar infectivity to the wild-type virus in the presence of human TRIM5 $\alpha$, and a virus with the SIVmac L4/5, L6/7, and G116E substitution (4/5SG116E6/7S) became more sensitive to human TRIM5 $\alpha$ (Figure 5B). On the other hand, the virus with the SIVmac L4/5 and L6/7 showed slightly impaired cleavage of p55 Gag precursors, although p24 mature CA proteins were clearly detected (Figure 6). However, the addition of G116E substitution did not facilitate the cleavage of Gag, and a small defect in Gag processing could only partially explain the attenuated growth of NL-4/5S6/7SvifS. Another possibility is that NL-4/5S6/7SvifS was restricted by a certain intrinsic restriction factor that was previously suggested to be present in human cells $[13,14]$, and that the adapted virus could escape from this restriction by G116E substitution, since the G116E was acquired through the adaptation in human cells. It is thus necessary to conduct further analysis to substantiate this unidentified restriction factor.

Although the G116E substitution occurred during long-term cultivation of human cells infected with NL-4/ 5S6/7SvifS, the viruses with G116E unexpectedly became resistant to CM TRIM5 $\alpha$-mediated restriction (Figures 4 and 5). Replacing the HIV-1 L6/7 (HNPPIP) of the CA with that of SIVmac239 (RQQNPIP) resulted in elongation of the loop by one amino acid, and it is reasonable to assume that the G116E substitution occurred to compensate the structural warp caused by the extended L6/7. This compensatory substitution occurred at the central position of the surface composed of L4/5 and L6/7, a structure considered to be important for TRIM5 $\alpha$ binding [24]. The amino acid substitution of $G$ with $E$ at the $116^{\text {th }}$ position caused an important change in the structure of the surface composed of L4/5 and L6/7 because G, which has no side chain, was replaced by E, which has a long, negatively charged side chain as shown in Figure 7. This change in the conformational structure of L4/5 and L6/7 might affect the interaction between the CA and TRIM5 $\alpha$. Alternatively, this single amino acid substitution might influence the configuration of surrounding loops by the changes in the side chain and polarity without directly involving the binding site of TRIM $5 \alpha$.

\section{Conclusion}

We succeeded in rescuing the impaired replicative capability of simian tropic HIV-1 NL-4/5S6/7SvifS and unexpectedly identified a single amino acid substitution in the CA that affects viral sensitivity to CM TRIM $5 \alpha$-mediated restriction. This finding will increase our understanding of the detailed molecular interactions between the CA and TRIM $5 \alpha$.

\section{Abbreviations \\ HIV-1: human immunodeficiency virus type 1; SIVmac: simian immunodefi- ciency virus isolated form macaque; CM: cynomolgus monkey; Rh: rhesus monkey; SHIV: HIV-1/SIV chimeric virus; CypA: cyclophilin A; TRIM: tripartite motif; CA: capsid; GFP: green fluorescence protein; VSV-G: vesicular stomatitis virus glycoprotein; SeV: Sendai virus; L4/5: a loop between a-helices 4 and 5; L6/7: a loop between a-helices 6 and 7}

\section{Competing interests}

The authors declare that they have no competing interests.

\section{Authors' contributions}

$A K$, and EEN performed the in vitro experiments; $K B$ performed computational modeling of CA protein; and AK, TS, KB and EEN wrote the paper.

\section{Acknowledgements}

The TRIM5a-KD Jurkat and Luci-siRNA Jurkat cells were kindly provided by Dr. Jeremy Luban. The authors wish to thank Ms. Setsuko Bandou and Ms. Noriko Teramoto for their helpful assistance. This work was supported by grants from the Health Science Foundation, the Ministry of Education, Culture, Sports, Science, and Technology, and the Ministry of Health, Labour and Welfare, Japan. 


\section{Author Details}

'Department of Viral Infections, Research Institute for Microbial Diseases, Osaka University, Osaka 565-0871, Japan and 2 Max Planck Institute for Informatics, Campus E1.4, 66123 Saarbrücken, Germany

Received: 8 February 2010 Accepted: 7 July 2010

Published: 7 July 2010

\section{References}

1. Shibata R, Sakai H, Kawamura M, Tokunaga K, Adachi A: Early replication block of human immunodeficiency virus type 1 in monkey cells. J Gen Virol 1995, 76(Pt 11):2723-2730.

2. Himathongkham S, Luciw PA: Restriction of HIV-1 (subtype B) replication at the entry step in rhesus macaque cells. Virology 1996, 219:485-488.

3. Hofmann W, Schubert D, LaBonte J, Munson L, Gibson S, Scammell J, Ferrigno P, Sodroski J: Species-specific, postentry barriers to primate immunodeficiency virus infection. J Virol 1999, 73:10020-10028.

4. Shibata R, Kawamura M, Sakai H, Hayami M, Ishimoto A, Adachi A: Generation of a chimeric human and simian immunodeficiency virus infectious to monkey peripheral blood mononuclear cells. J Virol 1991, 65:3514-3520.

5. Kamada K, Igarashi T, Martin MA, Khamsri B, Hatcho K, Yamashita T, Fujita M, Uchiyama T, Adachi A: Generation of HIV-1 derivatives that productively infect macaque monkey lymphoid cells. Proc Natl Acad Sci USA 2006, 103:16959-16964.

6. Goila-Gaur R, Strebel K: HIV-1 Vif, APOBEC, and intrinsic immunity. Retrovirology 2008, 5:51

7. Berthoux L, Sebastian S, Sokolskaja E, Luban J: Lv1 inhibition of human immunodeficiency virus type 1 is counteracted by factors that stimulate synthesis or nuclear translocation of viral cDNA. J Virol 2004 78:11739-11750.

8. Kootstra NA, Munk C, Tonnu N, Landau NR, Verma IM: Abrogation of postentry restriction of HIV-1-based lentiviral vector transduction in simian cells. Proc Natl Acad Sci USA 2003, 100:1298-1303.

9. Nakayama EE, Shingai Y, Kono K, Shioda T: TRIM5alpha-independent anti-human immunodeficiency virus type 1 activity mediated by cyclophilin A in Old World monkey cells. Virology 2008, 375:514-520.

10. Van Damme N, Goff D, Katsura C, Jorgenson RL, Mitchell R, Johnson MC Stephens EB, Guatelli J: The interferon-induced protein BST-2 restricts HIV-1 release and is downregulated from the cell surface by the viral Vpu protein. Cell Host Microbe 2008, 3:245-252.

11. Neil SJ, Zang T, Bieniasz PD: Tetherin inhibits retrovirus release and is antagonized by HIV-1 Vpu. Nature 2008, 451:425-430

12. Stremlau M, Owens CM, Perron MJ, Kiessling M, Autissier P, Sodroski J: The cytoplasmic body component TRIM5alpha restricts HIV-1 infection in Old World monkeys. Nature 2004, 427:848-853.

13. Luban J: Cyclophilin A, TRIM5, and resistance to human immunodeficiency virus type 1 infection. J Viro/ 2007, 81:1054-1061.

14. Towers GJ: The control of viral infection by tripartite motif proteins and cyclophilin A. Retrovirology 2007, 4:40.

15. Hatziioannou T, Ambrose Z, Chung NP, Piatak M Jr, Yuan F, Trubey CM, Coalter V, Kiser R, Schneider D, Smedley J, Pung R, Gathuka M, Estes JD, Veazey RS, KewalRamani VN, Lifson JD, Bieniasz PD: A macaque model of HIV-1 infection. Proc Natl Acad Sci USA 2009, 106:4425-4429.

16. Kamada K, Yamashita T, Hatcho K, Adachi A, Nomaguchi M: Evasion from CypA- and APOBEC-mediated restrictions is insufficient for HIV-1 to efficiently grow in simian cells. Microbes Infect 2009, 11:164-171.

17. Reymond A, Meroni G, Fantozzi A, Merla G, Cairo S, Luzi L, Riganelli D, Zanaria E, Messali S, Cainarca S, Guffanti A, Minucci S, Pelicci PG, Ballabio A: The tripartite motif family identifies cell compartments. EMBO J 2001 20:2140-2151

18. Nakayama EE, Miyoshi H, Nagai Y, Shioda T: A specific region of 37 amino acid residues in the SPRY (B30.2) domain of African green monkey TRIM5alpha determines species-specific restriction of simian immunodeficiency virus SIVmac infection. J Virol 2005, 79:8870-8877.

19. Ohkura S, Yap MW, Sheldon T, Stoye JP: All three variable regions of the TRIM5alpha B30.2 domain can contribute to the specificity of retrovirus restriction. J Virol 2006, 80:8554-8565.

20. Li Y, Li X, Stremlau M, Lee M, Sodroski J: Removal of arginine 332 allows human TRIM5alpha to bind human immunodeficiency virus capsids and to restrict infection. J Virol 2006, 80:6738-6744.
21. Sawyer SL, Wu LI, Emerman M, Malik HS: Positive selection of primate TRIM5alpha identifies a critical species-specific retroviral restriction domain. Proc Natl Acad Sci USA 2005, 102:2832-2837.

22. Perez-Caballero D, Hatziioannou T, Yang A, Cowan S, Bieniasz PD: Human tripartite motif 5 alpha domains responsible for retrovirus restriction activity and specificity. J Virol 2005, 79:8969-8978.

23. Song H, Nakayama EE, Yokoyama M, Sato H, Levy JA, Shioda T: A single amino acid of the human immunodeficiency virus type 2 capsid affects its replication in the presence of cynomolgus monkey and human TRIM5alphas. J Virol 2007, 81:7280-7285.

24. Kuroishi A, Saito A, Shingai Y, Shioda T, Nomaguchi M, Adachi A, Akari H, Nakayama EE: Modification of a loop sequence between alpha-helices 6 and 7 of virus capsid (CA) protein in a human immunodeficiency virus type 1 (HIV-1) derivative that has simian immunodeficiency virus (SIVmac239) vif and CA alpha-helices 4 and 5 loop improves replication in cynomolgus monkey cells. Retrovirology 2009, 6:70.

25. Adachi A, Gendelman HE, Koenig S, Folks T, Willey R, Rabson A, Martin MA: Production of acquired immunodeficiency syndrome-associated retrovirus in human and nonhuman cells transfected with an infectious molecular clone. J Virol 1986, 59:284-291.

26. Miyoshi H, Takahashi M, Gage FH, Verma IM: Stable and efficient gene transfer into the retina using an HIV-based lentiviral vector. Proc Natl Acad Sci USA 1997, 94:10319-10323.

27. Miyoshi H, Blomer U, Takahashi M, Gage FH, Verma IM: Development of a self-inactivating lentivirus vector. J Viro/ 1998, 72:8150-8157.

28. Kono K, Song H, Shingai Y, Shioda T, Nakayama EE: Comparison of antiviral activity of rhesus monkey and cynomolgus monkey TRIM5alphas against human immunodeficiency virus type 2 infection. Virology 2008, 373:447-456.

29. Tang C, Ndassa Y, Summers MF: Structure of the N-terminal 283-residue fragment of the immature HIV-1 Gag polyprotein. Nat Struct Biol 2002, 9:537-543.

30. Eswar N, Webb B, Marti-Renom MA, Madhusudhan MS, Eramian D, Shen MY, Pieper U, Sali A: Comparative protein structure modeling using MODELLER. Curr Protoc Protein Sci 2007, Chapter 2:. Unit 29

31. Maegawa H, Nakayama EE, Kuroishi A, Shioda T: Silencing of tripartite motif protein (TRIM) 5alpha mediated anti-HIV-1 activity by truncated mutant of TRIM5alpha. J Virol Methods 2008, 151:249-256.

doi: $10.1186 / 1742-4690-7-58$

Cite this article as: Kuroishi et al., A single amino acid substitution of the human immunodeficiency virus type 1 capsid protein affects viral sensitivity to TRIM5 a Retrovirology 2010, 7:58

\section{Submit your next manuscript to BioMed Centra and take full advantage of:}

- Convenient online submission

- Thorough peer review

- No space constraints or color figure charges

- Immediate publication on acceptance

- Inclusion in PubMed, CAS, Scopus and Google Scholar

- Research which is freely available for redistribution 